\title{
Limit on Lorentz-Invariance- and CPT-Violating Neutron Spin Interactions Using a ${ }^{3} \mathrm{He}^{129} \mathrm{Xe}$ Comagnetometer
}

\author{
F. Allmendinger and U. Schmidt \\ Physikalisches Institut, Ruprecht-Karls-Universität, 69120 Heidelberg, Germany \\ W. Heil, S. Karpuk, Yu. Sobolev and K. Tullney \\ Institut für Physik, Johannes Gutenberg-Universität, 55099 Mainz, Germany
}

Published 29 February 2016

\begin{abstract}
We performed a search for a Lorentz-invariance- and CPT-violating coupling of the ${ }^{3} \mathrm{He}$ and ${ }^{129} \mathrm{Xe}$ nuclear spins to posited background fields. Our experimental approach is to measure the free precession of nuclear spin polarized ${ }^{3} \mathrm{He}$ and ${ }^{129} \mathrm{Xe}$ atoms using SQUIDs as detectors. As the laboratory reference frame rotates with respect to distant stars, we look for a sidereal modulation of the Larmor frequencies of the co-located spin samples. As a result we obtain an upper limit on the equatorial component of the background field $\tilde{b}_{\perp}^{n}<8.4 \cdot 10^{-34} \mathrm{GeV}(68 \%$ C.L.). This experiment is currently the most precise test of spin anisotropy due to the excellent long spin-coherence time.
\end{abstract}

\section{Introduction and Experimental Setup}

In the context of the Standard-Model Extension (SME),${ }^{1-3}$ couplings of the neutron or proton spin $\boldsymbol{\sigma}^{\boldsymbol{n}, \boldsymbol{p}}$ to relic background fields $\tilde{\boldsymbol{b}}^{\boldsymbol{n}, \boldsymbol{p}}$ are discussed. The background fields have distinct directions in space and correspond to preferred spin directions. These couplings with the potential $V=\tilde{\boldsymbol{b}}^{\boldsymbol{n}, \boldsymbol{p}} \cdot \boldsymbol{\sigma}^{\boldsymbol{n}, \boldsymbol{p}}$ are purely non-magnetic, but change the energy levels of spins in a magnetic field, which can be detected by changes in the Larmor frequency of precessing spins.

Nuclear spin clocks, based on the detection of free spin precession of gaseous nuclear polarized ${ }^{3} \mathrm{He}$ and ${ }^{129} \mathrm{Xe}$ atoms with $\mathrm{LT}_{C}$ SQUIDs as low-noise magnetic flux detectors are used as ultra-sensitive probe for nonmagnetic spin interactions, since the magnetic interaction (Zeeman term) drops out in the case of co-located spin samples (comagnetometry). Measurements of uninterrupted precession of $T \sim 1$ day can

*Corresponding author: allmendinger@physi.uni-heidelberg.de.

This is an Open Access article published by World Scientific Publishing Company. It is distributed under the terms of the Creative Commons Attribution 3.0 (CC-BY) License. Further distribution of this work is permitted, provided the original work is properly cited. 
be achieved at the present stage of investigation due to long spin-coherence times. The principle of measurement is to search for sidereal variations of the precession frequency of co-located spin species while the Earth and hence the laboratory reference frame rotates with respect to distant stars.

To give a short overview of the setup (details are given in Ref. ${ }^{4}$ ): The two polarized gas species (and $\mathrm{N}_{2}$ as a buffer gas) are filled into a low-relaxation spherical glass cell with radius $R=5 \mathrm{~cm}$. Typically, the optimum conditions in terms of long transverse relaxation times $\left(T_{2}^{*}\right)$ and high Signal-to-Noise Ratio are met at a gas mixture with pressures of $p_{H e}=3$ mbar, $p_{X e}=5$ mbar, $p_{N 2}=25$ mbar. The cell is positioned in a homogeneous static magnetic field (about $400 \mathrm{nT}$ ) that is generated by Helmholtz coils mounted inside the strongly magnetically shielded room BMSR-2 at the Physikalisch-Technische Bundesanstalt in Berlin. At that field strength, the Larmor frequencies of ${ }^{3} \mathrm{He}$ and ${ }^{129} \mathrm{Xe}$ are about $\omega_{\mathrm{He}} \approx 2 \pi \cdot 13 \mathrm{~Hz}$ and $\omega_{X e} \approx 2 \pi \cdot 4.7 \mathrm{~Hz}$, respectively. To measure these precession frequencies very precisely, low-noise low-temperature DC-SQUID gradiometers are used as magnetic flux detectors. Due to the very low field gradients in the order of $\mathrm{pT} / \mathrm{cm}$ at the location of the cell, the transverse relaxation times reached $T_{2}^{*}=8.5 \mathrm{~h}$ for ${ }^{129} \mathrm{Xe}$ and up to $T_{2}^{*}=100 \mathrm{~h}$ for ${ }^{3} \mathrm{He} .{ }^{4}$ The measured signal amplitudes at the beginning of the measurement were up to $A_{\mathrm{He}}=20 \mathrm{pT}$ and $A_{\mathrm{Xe}}=8 \mathrm{pT}$ for ${ }^{3} \mathrm{He}$ and ${ }^{129} \mathrm{Xe}$, respectively. The noise level (combination of four gradiometers) was $\rho=3 \mathrm{fT} / \sqrt{\mathrm{Hz}}$. Due to the long spin-coherence time and the high initial Signal-to-Noise Ratio, the spin precession could be monitored for more than one day, which improves the sensitivity remarkably (see below).

\section{Data Evaluation and Results}

To be sensitive to tiny nonmagnetic interactions, one has to consider the weighted difference of the respective Larmor frequencies of the co-located spin samples, or the corresponding time integral (weighted phase difference), which are defined by

$$
\Delta \omega=\omega_{\mathrm{He}}-\frac{\gamma_{\mathrm{He}}}{\gamma_{\mathrm{Xe}}} \omega_{\mathrm{Xe}} \text { and } \Delta \Phi=\Phi_{\mathrm{He}}-\frac{\gamma_{\mathrm{He}}}{\gamma_{\mathrm{Xe}}} \Phi_{\mathrm{Xe}}
$$

In doing so, magnetic field fluctuations are canceled, i.e. in principle $\Delta \omega=0$ and $\Delta \Phi=$ const. if there are no further interactions. However, on a closer look, $\Delta \Phi$ is not constant in time, as higher order effects have to be take into account. These can be parameterized by

$$
\Delta \Phi(t)=c_{0}+c_{1} t+E_{\mathrm{He}} e^{-t / T_{2, \mathrm{He}}^{*}}+E_{\mathrm{Xe}} e^{-t / T_{2, \mathrm{Xe}}^{*}}+F_{\mathrm{He}} e^{-2 t / T_{2, \mathrm{He}}^{*}}+F_{\mathrm{Xe}} e^{-2 t / T_{2, \mathrm{Xe}}^{*}} .
$$

The linear contribution stems from Earth's rotation (i.e. the rotation of the SQUID detectors with respect to the precessing spins) and from chemical shift (diamagnetic shielding: the electron shells shield the nuclei against the external magnetic guiding field). The four exponential terms account for the Ramsey-Bloch-Siegert shift. ${ }^{5,6}$ 
These effects are discussed in Ref.. ${ }^{4}$ Finally, the function in Eq. (2) together with the appropriate parameterization of the Lorentz-invariance-violating effect - in this case a sidereal modulation $\propto \tilde{b} \cdot \sin \left(\Omega_{S} \cdot t+\varphi_{0}\right)$ - is fitted to the combined weighted phase difference data of all measurement runs ( 7 in total). The resulting estimate on sidereal modulation is compatible with zero within the correlated and uncorrelated uncertainties and can be expressed as an upper limit on the magnitude of the hypothetical background field:

$$
\tilde{b}_{\perp}^{\mathrm{n}}<6.7 \cdot 10^{-34} \mathrm{GeV}(68 \% \text { C.L. })
$$

\section{Corresponding Limits on Proton Interactions}

In Ref., ${ }^{9}$ Y. Stadnik and V. Flambaum calculated the neutron and proton contributions to the nuclear spin for several nuclei of experimental interest. They showed that the ${ }^{3} \mathrm{He}$ and ${ }^{129} \mathrm{Xe}$ system is also very sensitive to the coupling of the proton spin to a hypothetical background field.

The different contributions to the nuclear magnetic moment $\mu$ can be expressed as

$$
\frac{\mu}{\mu_{N}}=g_{p}<s_{p}^{z}>+g_{n}<s_{n}^{z}>+g_{l}<l_{p}^{z}>
$$

with the nuclear magneton $\mu_{N}$, the expectation values of the total neutron and proton spins $\left\langle s_{n}^{z}>\right.$ and $\left\langle s_{p}^{z}>\right.$, and the expectation value of the total proton orbital momentum $\left\langle l_{p}^{z}\right\rangle$. The gyromagnetic factors are $g_{p}=5.586, g_{n}=-3.826$ and $g_{l}=1$.

Taking the experimental values of the magnetic moments of the nuclei (and neglecting spin-orbit interaction), Stadnik and Flambaum calculated the proton and neutron spin contributions. In particular with $\left\langle l_{p}^{z}\right\rangle=0$ for ${ }^{3} \mathrm{He}$ and ${ }^{129} \mathrm{Xe}$ :

$$
\begin{aligned}
& <s_{p}^{z}>_{H e}=0.000 \\
& <s_{n}^{z}>_{H e}=0.500
\end{aligned}
$$

and

$$
\begin{aligned}
& <s_{p}^{z}>_{X e}=0.135 \\
& <s_{n}^{z}>_{X e}=0.365
\end{aligned}
$$

In words, for ${ }^{3} \mathrm{He}$ the Schmidt model is valid and the magnetic moment of ${ }^{3} \mathrm{He}$ is determined by the valence neutron. However, for ${ }^{129} \mathrm{Xe}$ the proton contribution is large. Therefore, the ${ }^{3} \mathrm{He}^{129} \mathrm{Xe}$ comagnetometer is also sensitive to the proton interaction parameters of the SME: ${ }^{9}$

$$
\begin{aligned}
& \tilde{b}_{X}^{\mathrm{n}}+0.74 \cdot \tilde{b}_{X}^{\mathrm{p}}=(7.1 \pm 8.2) \cdot 10^{-34} \mathrm{GeV} \\
& \tilde{b}_{X}^{\mathrm{n}}+0.74 \cdot \tilde{b}_{Y}^{\mathrm{p}}=(5.0 \pm 10.8) \cdot 10^{-34} \mathrm{GeV}
\end{aligned}
$$


with the corresponding upper limit of the equatorial component $\tilde{b}_{\perp}^{\mathrm{p}}$ of the background tensor field interacting with the spin of the bound proton:

$$
\tilde{b}_{\perp}^{\mathrm{p}}<1.6 \cdot 10^{-33} \mathrm{GeV}(68 \% \text { Confidence Level }) .
$$

This value improves the previously best limit by a factor 35 .

\section{Frequency Stability}

The benefit of long spin-coherence times can be explained on the basis of the Cramer-Rao Lower Bound (CRLB). The CRLB gives the minimum variance of an unbiased estimator of a deterministic parameter. In this case, the sensitivity of frequency measurements depends on the measurement time with coherent spin precession $T$, the signal amplitude at the beginning of the measurement $A$ decaying with a time constant $T_{2}^{*}$ and the noise level $\rho$ (assuming white noise). ${ }^{7}$ The frequency uncertainty is:

$$
\sigma_{f} \geq \frac{\sqrt{3}}{\pi} \frac{\rho}{A} \cdot T^{-3 / 2} \cdot \sqrt{C\left(T, T_{2}^{*}\right)}
$$

with the dimensionless factor $C\left(T, T_{2}^{*}\right)$ accounting for the exponential decay of the Signal-to-Noise Ratio. The remarkable result is that the frequency uncertainty decreases with $T^{-3 / 2}$ for white (Gaussian) noise. The Allan Standard Deviation (ASD) is the appropriate measure to study the temporal characteristics of the ${ }^{3} \mathrm{He}-$ ${ }^{129} \mathrm{Xe}$ comagnetometer with respect to phase or frequency determination. The ASD method is an established analysis technique for studying the low-frequency component of a time series and is a measure of phase and frequency stability of clocks and oscillators. In the case of the ${ }^{3} \mathrm{He}-{ }^{129} \mathrm{Xe}$ comagnetometer, one compares the ${ }^{3} \mathrm{He}$ precession frequency to the "scaled" ${ }^{129} \mathrm{Xe}$ precession frequency. These two oscillator frequencies are supposed to be the same if all deterministic phase shifts (chemical shift, Earth's rotation, Ramsey-Bloch-Siegert shift etc.) have been incorporated correctly. The behavior of the frequency uncertainty in the ASD plot is shown in Fig. 1 (black symbols) for two different runs. With increasing integration times $\tau$ the uncertainty in frequency decreases with $\sigma_{\mathrm{ASD}} \propto \tau^{-\frac{3}{2}}$ as expected by the CRLB in Eq. (9). This indicates the presence of pure white (Gaussian) noise. In other words: All deterministic phase shifts (chemical shift, Earth's rotation, Ramsey-Bloch-Siegert shift etc.) have been incorporated correctly. This has been tested for all measurement runs with different experimental conditions (e.g. different sample cells, partial pressures, magnetic field directions, $T_{2}^{*}$ ) and the ASD plot shows the expected behavior, leading to the conclusion that the fit model in Eq. (2) describes the behavior of the comagnetometer correctly. An incorrect fit model would immediately lead to a deviation from the CRLB power law. This is also demonstrated in Fig. 1 (gray symbols) showing the ASD as a function of $\tau$ for the residuals of a fit model where the $F_{\mathrm{He}}$, Xe terms (called "cross-talk") of Eq. (2) are omitted. For run 6 (the run with largest cross-talk effect), the large deviation 

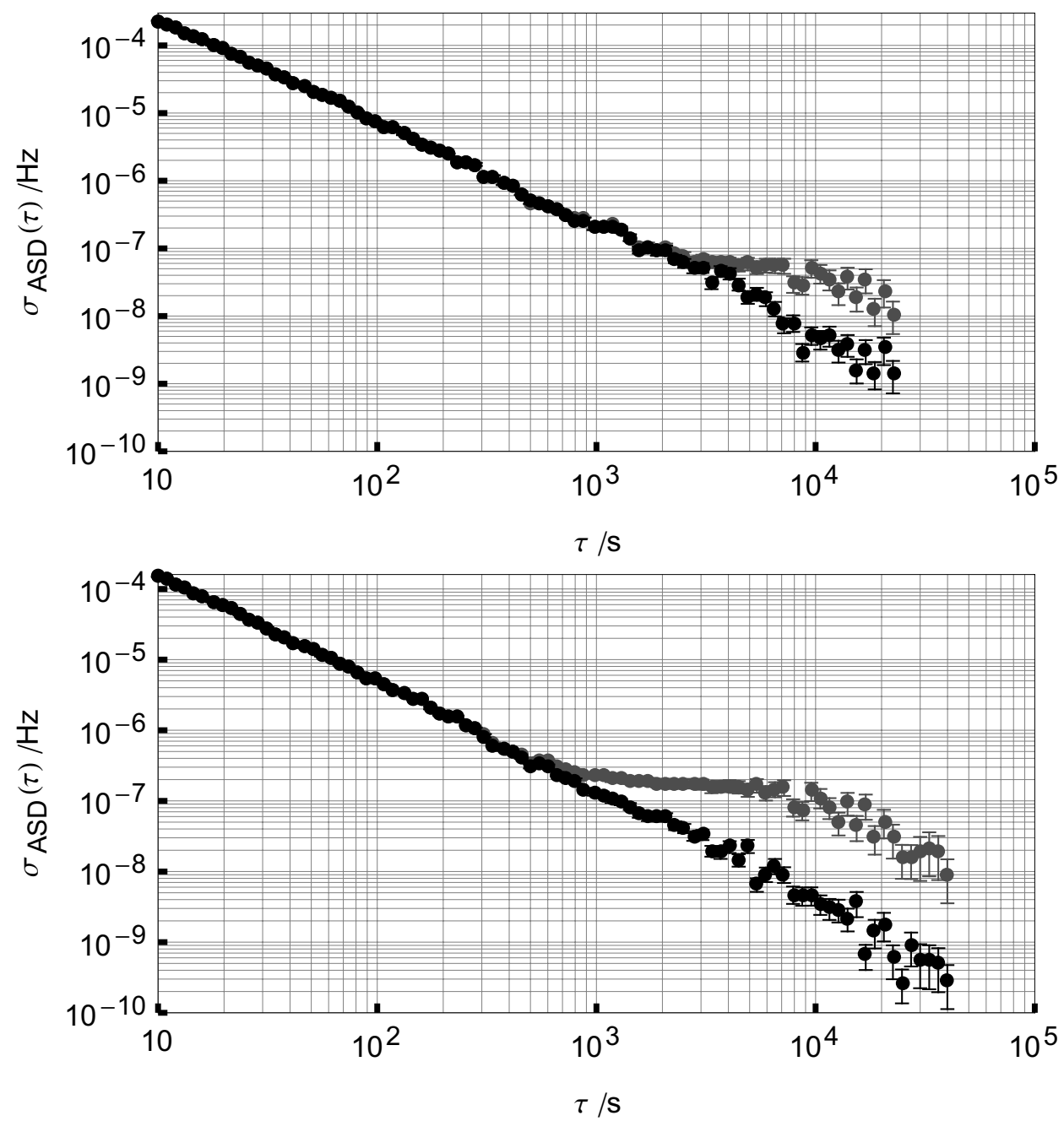

Fig. 1. ASD plot of the residual frequency noise of two independent runs (Top: Run 3, Bottom: Run 6). Black symbols: ASD for the correct fit model (Eq. (2)). With increasing integration times $\tau$ the uncertainty in frequency decreases as $\sigma \propto \tau^{-\frac{3}{2}}$ indicating the presence of white (Gaussian) noise. Gray symbols: ASD for an incorrect fit model (omitting the Cross-Talk term). For run 6 (the run with largest cross-talk effect), the large deviation from the CRLB power law above a few hundred seconds is impressive. For run 3 (the run with the smallest cross-talk effect) the deviation in the ASD plot is smaller.

from the CRLB power law above $\tau \approx 500 \mathrm{~s}$ is impressive. For run 3 (the run with the smallest cross-talk effect) the deviation in the ASD plot is smaller.

\section{Conclusion and Outlook}

Freely precessing gaseous, nuclear polarized ${ }^{3} \mathrm{He}$ and ${ }^{129} \mathrm{Xe}$ samples can be used as ultra-sensitive probe for nonmagnetic spin interactions, since the magnetic 
interaction (Zeeman term) drops out in the case of co-located spin samples. Long spin-coherence times are highly beneficial as the uncertainty in frequency estimation decreases with $T^{-3 / 2}$ for Gaussian noise according to the CRLB. The ASD method can be used to verify the CRLB power law and thereby rule out the presence of further noise sources (i.e. further deterministic frequency shifts). With a similar setup, upper limits on interactions mediated by axion-like particles were obtained. ${ }^{8}$ The next challenging step is to apply this method in the search for an electric dipole moment of ${ }^{129} \mathrm{Xe}$ : A permanent EDM $\boldsymbol{d}$ of a fundamental or composite particle must be aligned parallel to the spin, as the spin is the only available vector for an eigenstate of the isolated particle. Thus, for a magnetic guiding field aligned along the z-direction, the Hamiltonian has the form $H=-\mu \cdot B_{0}-d \cdot E_{z}$, with the corresponding frequency shift $\delta \omega_{\mathrm{EDM}}=\frac{2}{\hbar} d \cdot E_{z}$. By varying the z-component of the electric field $E_{z}$, the frequency shift is modulated correspondingly. As mentioned before, the principle of comagnetometry is applied to become insensitive to drifts of the magnetic guiding field. Subsequently, a non-zero EDM will manifest in a modulation of the weighted phase difference, and the corresponding value $d$ can be extracted.

\section{References}

1. V. A. Kostelecký and S. Samuel, Phys. Rev. D 39, 683 (1989).

2. D. Colladay and V. A. Kostelecký, Phys. Rev. D 58, 116002 (1998).

3. V. A. Kostelecký and C. D. Lane, Phys. Rev. D 60, 116010 (1999).

4. F. Allmendinger et. al, Phys. Rev. Lett. 112, 110801 (2014).

5. F. Bloch and A. Siegert, Phys. Rev. 57, 522 (1940).

6. N. F. Ramsey, Phys. Rev. 100, 1191 (1955).

7. W. Heil et al., Ann. Phys. (Berlin) 525, 539 (2013).

8. K. Tullney et al., Phys. Rev. Lett. 111, 100801 (2013).

9. Y. V. Stadnik and V. V. Flambaum, arXiv:1408.2184. 